\title{
Measurement of cosmic-ray proton + helium spectrum with DAMPE
}

\author{
Ivan De Mitri, ${ }^{1,2}$ Giovanni Marsella, ${ }^{3,4}$ Guillermo Torralba Elipe, ${ }^{1,2}$ Ines Valino, ${ }^{1,2}$ \\ Zhaomin Wang ${ }^{* 1,2}$ on behalf of DAMPE collaboration! \\ ${ }^{1}$ Gran Sasso Science Institute, L'Aquila, Italy \\ ${ }^{2}$ Istituto Nazionale di Fisica Nucleare, Laboratori Nazionali del Gran Sasso, Assergi (AQ), Italy \\ ${ }^{3}$ Dipartimento di Matematica e Fisica "E. De Giorgi", Universita del Salento, Lecce, Italy \\ ${ }^{4}$ Istituto Nazionale di Fisica Nucleare Sezione di Lecce, Lecce, Italy \\ E-mail: zhaomin.wangegssi.it
}

The DAMPE (Dark Matter Particle Explorer) is a powerful space-borne experiment for direct detection of high-energy cosmic rays, electrons and gamma rays. While it was designed mainly to search for dark matter signatures in the electron and photon spectra, DAMPE distinguishes itself also by its capability to study the cosmic-ray fluxes of the primary species with unprecedented resolution (better than $40 \%$ at $800 \mathrm{GeV}$ ) in an energy range from few tens of $\mathrm{GeV}$ up to $100 \mathrm{TeV}$. The selection of the incoming particle is done by means of the Plastic Scintillator Detector (PSD), whose signal is proportional to the square of its electric charge. Selecting the $\mathrm{H}+\mathrm{He}$ samples has the advantage of almost no background and very high purity. Here we present the preliminary results of the measurement of the proton + helium energy spectrum measured from $50 \mathrm{GeV}$ to 10 $\mathrm{TeV}$. The observed spectral features are also discussed and the results are compared with other measurements.

36th International Cosmic Ray Conference -ICRC2019-

July 24th - August 1st, 2019

Madison, WI, U.S.A.

\footnotetext{
* Speaker.

${ }^{\dagger}$ For collaboration list see PoS(ICRC2019) 1177
} 


\section{Introduction}

Various observations of galactic Cosmic Ray (CR) spectra have revealed a deviation from the single power law behaviour, showing a spectral hardening at $\sim 250 \mathrm{GeV} / \mathrm{n}$ in the observation of PAMELA [1], AMS-02 [2], CALET [3] and ATIC-02 [4]. At larger energies present data (coming from calorimetric balloon-borne experiments) might suggest a spectral softening. For instance the CREAM-III [5] observation shows a softening in the nuclei spectrum at $\sim 10 \mathrm{TeV} / \mathrm{n}$. But both statistic and systematic uncertainties prevent any conclusion. Thanks to the thick fine-grained calorimeter and relatively large acceptance, DAMPE (DArk Matter Particle Explorer) can cover this energy region up to $100 \mathrm{TeV}$ with good statistics and limited systematic uncertainties.

DAMPE is a powerful calorimetric-type space-borne experiment for direct detection of high energy CR nuclei, electrons and gamma rays. DAMPE is able to measure the CR proton $(\mathrm{H})$ and Helium (He) nuclei spectra, the main components of CR, up to $100 \mathrm{TeV}$ with unprecedented energy resolution [6]. DAMPE was launched on 17th December 2015, and has been smoothly collecting the data since then. With its large acceptance, the CR statistics from DAMPE is sufficient enough to reveal the precise features of CR nuclei spectra.

In general, the $\mathrm{H}$ and $\mathrm{He}$ spectra are studied separately. However, combining the $\mathrm{H}$ and $\mathrm{He}$ together in a single spectrum measurement has certain strong advantages. The $\mathrm{H}+\mathrm{He}$ spectrum has very low contamination (less than $0.1 \%$ ) and can be regarded as a crosscheck for $\mathrm{H}$ and $\mathrm{He}$ singular spectra. Besides, since DAMPE is able to measure CRs up to $100 \mathrm{TeV}$ and higher energies (with large accumulated statistics), the $\mathrm{H}+\mathrm{He}$ spectrum obtained by DAMPE provides an important link between direct $\mathrm{CR}$ measurements and indirect ones which, in general, cannot identify $\mathrm{H}$ and $\mathrm{He}$ components separately.

\section{DAMPE Instrument}

DAMPE system is composed of four sub-detectors. The Plastic Scintillator Detector (PSD) [7] that includes two planes of Plastic Scintillator bars is used to measure the charge of entering cosmic rays. The Silicon Tungsten Tracker (STK) [8], composed of six double layers silicon detectors, is responsible for reconstructing the particle tracks. The bismuth germanate (BGO) calorimeter [9] that consists of 14 layers of BGO bars allows to reconstruct the energy of entering cosmic rays. The Neutron Detector (NUD) formed by four blocks of boron doped plastic scintillator provides an additional electron-hadron discrimination. More details about DAMPE detectors can be found in $[6,7,8,9,10]$.

\section{The data sample}

In this analysis, the data used covers the time period from the 1st January 2016 up to the 30th March 2019. Note that there is some dead time during the orbit period of DAMPE. Since DAMPE passes through the so-called South Atlantic Anomaly (SAA) [11] region 6-7 times per day (a 4.5\% of the total flying time), the data collected in this area are rejected. The responding time of the electronics (about $3 \mathrm{~ms}$ per each triggered event) must be also accounted for, which corresponds to a $18 \%$ of the total time given the DAMPE trigger rate [12]. Finally, the daily on-orbit calibration 
and the monthly electronics-linearity calibration must be removed (a 1.8\% of the total orbit time). As a result, the total exposure time amounts to $7.7 \times 10^{7} \mathrm{~s}$, which is equivalent to 892 days.

To guarantee a high-quality data set for the measurement of the energy spectrum, a set of selection cuts are applied on data. Those are described briefly below.

\subsection{Pre-selection}

The pre-selection cuts, based on the BGO measurement, guarantee that the showers of entering particles are fully contained inside the BGO calorimeter and not influenced by the geomagnetic cutoff [13].In particular, these cuts include: 1) The reconstructed energy in the BGO of each event is larger than $20 \mathrm{GeV}$ to remove the effect of the geomagnetic cutoff; 2) The reconstructed track of the particle in the BGO must be fully contained in the calorimeter; 3) Events with more than 35\% of total energy deposited in a single BGO layer are rejected; 4) For the top three layers of the BGO, the BGO bar with the highest energy deposition must not be at the edge of the layer.

\subsection{Track reconstruction and selection}

Due to pre-showering of cosmic ray and back scattering of particles from the BGO, normally more than one track is reconstructed in the event. For each event, the selection of the best track is done by searching the best match between the STK and the BGO, and between the PSD and the STK. This is done by applying the following cuts: 1) the track is reconstructed with a $\chi^{2} / n d o f$ lower than $25 ; 2$ ) the track should have at least one cluster in one of the layers of the first plane of the STK to ensure a better charge measurement; 3) the angle between the STK track and BGO track must be less than $25^{\circ}$; 4) the distance between the projection of the STK track and the BGO track on the first layer of BGO must be less than $60 \mathrm{~mm}$; 5) the distance between the projection of the BGO track on the first layer of the STK and the STK hit position must be less than $200 \mathrm{~mm} ; 6$ ) the distance between the projection of the STK track on the first layer of BGO and the position of the BGO bar with maximum energy deposition must be less than $30 \mathrm{~mm}$;) the projection of the STK track on the first layer of the PSD must not be at the edge of the PSD; 8) The PSD bars passed through by the track must have energies higher than $0.5 \mathrm{MeV}$.

\subsection{Trigger selection and shower development}

In addition, events are required to activate the High Energy Trigger (HET) of the DAMPE trigger system [6] and not to enter from the bottom of the detector. The latter condition requires that the sum of the energy deposition in the first and second layers of BGO is less than the sum of the energy in the third and fourth layers of BGO.

\subsection{PSD charge selection}

In this analysis, the selection of the protons and helium is based on the particle charge measurement in the PSD. After the corrections for the light attenuation [16] and for the incidence angle in the PSD bars [17], the energy deposit is proportional to $Z^{2}$, and is expected to be about $2 \mathrm{MeV}$ for protons. Since each layer of PSD can measure the particle charge independently, we combine the two measurements together and use the average value (referred as $E_{\mathrm{PSD}}$ ) as the charge reference to select the particles. 
The combined $E_{\mathrm{PSD}}$ distribution of PSD for protons and helium for one BGO energy bin is presented on the left of Fig.1, where the peaks of proton and helium can be clearly seen. The peaks are fitted with the convolution function of Gaussian and Landau distributions. On the right of the Fig.1, the Most Probable Values $(M P V)$ of the fitting results as a function of BGO energy are shown. The shadow region represents the PSD selection range for the $\mathrm{H}+\mathrm{He}$ spectrum analysis. The upper boundary equals $M P V_{\mathrm{He}}+6 \sigma_{\mathrm{He}}$, where $M P V_{\mathrm{He}}$ represents the MPV of helium fitting results, while $\sigma_{\mathrm{He}}$ is the square root of the quadratic sum of the Gaussian sigma and Landau sigma. The lower boundary equals $M P V_{\mathrm{H}}-3 \sigma_{\mathrm{H}}$.
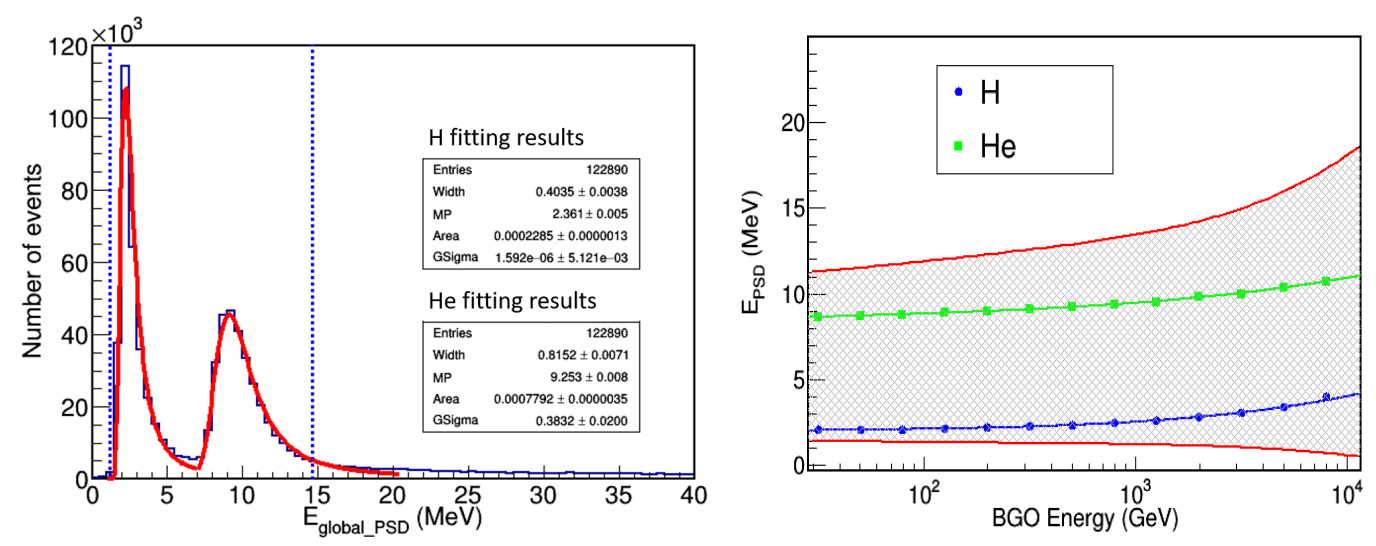

Figure 1: Left: the combined $E_{\mathrm{PSD}}$ distribution of PSD for $\mathrm{H}$ and He with BGO energy $398.1 \mathrm{GeV}-630 \mathrm{GeV}$. The red lines represent the fitting functions. The blue dash lines mark the PSD selection range. Right: the PSD selection range as function of BGO energy for $\mathrm{H}+\mathrm{He}$ spectrum analysis. The green squares represent the $\mathrm{He}$ data, while the blue points represent the $\mathrm{H}$ data. Both $\mathrm{H}$ and $\mathrm{He}$ data are fitted with fourth-order polynomial function. The shadow region shows the PSD selection range.

\section{Acceptance}

The effective acceptance of DAMPE system is defined as the geometric acceptance multiplying the efficiencies of all the selection cuts. It is derived from the Monte Carlo (MC) data as:

$$
A_{\mathrm{acc}, i}=A_{\mathrm{gen}} \times \frac{N_{\mathrm{pass}, i}}{N_{\mathrm{gen}, i}},
$$

where $A_{\mathrm{gen}}$ is the geometric factor used for generating the MC data. $N_{\mathrm{gen}, i}$ and $N_{\mathrm{pass}, i}$ are the number of generated events and those that pass the selections. The acceptances for each set of cuts are presented in Fig. 2. Besides the mentioned selections in the previous section, there are also other cuts to ensure the purity of the selected samples that include: the limitation on the signal difference between the two PSD layers, a more stringent STK-BGO track match, and electron removal. The final effective acceptance for $\mathrm{H}+\mathrm{He}$ is around $0.05 \mathrm{~m}^{2} \mathrm{sr}$ at $10^{4} \mathrm{GeV}$.

\section{Unfolding of the detector response}

Since the nuclear interaction length in the BGO calorimeter is $\sim 1.6$, the energy deposition for $\mathrm{H}$ and $\mathrm{He}$ nuclei in the $\mathrm{BGO}$ is only $35 \%-40 \%$ [6]. A correction must be applied to account for 


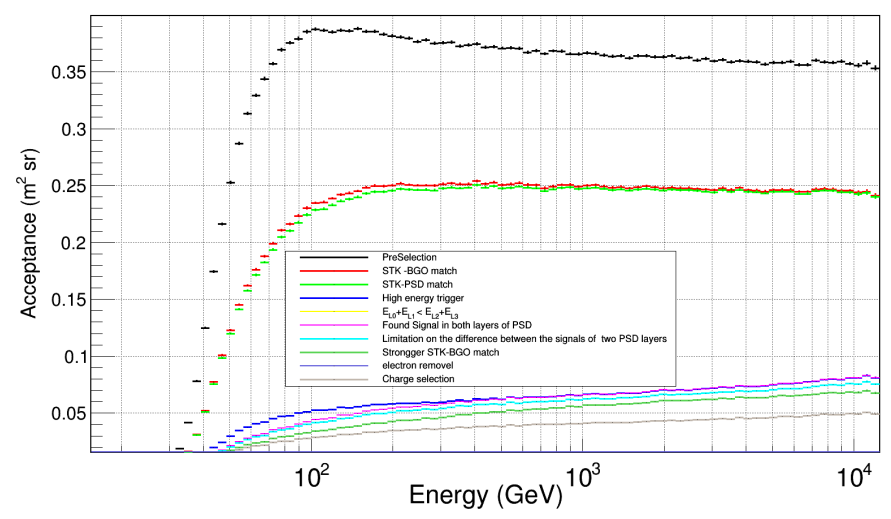

Figure 2: The acceptances for each set of selection cuts.

the effect of the detector resolution in the energy determination of $\mathrm{H}+\mathrm{He}$ candidates, responsible for a bin-to-bin event migration in the measurement of the energy spectrum. To correct for this, an unfolding method based on the Bayes' Theorem [18] is performed. The detector effects are estimated using MC simulations. Fig. 3 shows the response function, the so-called resolution matrix, of the DAMPE detector as obtained with $\mathrm{MC} \mathrm{H}+\mathrm{He}$ sample.

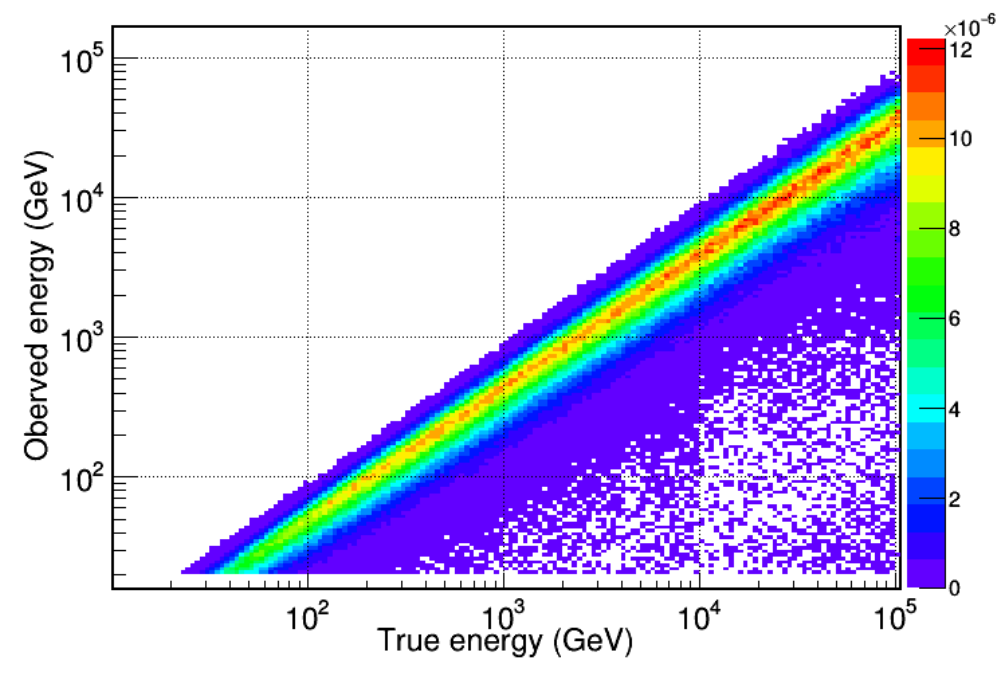

Figure 3: The resolution matrix obtained from $\mathrm{MC}$ selected $\mathrm{H}+\mathrm{He}$ sample

\section{Systematic uncertainties}

In this analysis, there are four sources of systematic uncertainties: the track selection efficiency; the High Energy Trigger (HET) efficiency; the charge reconstruction efficiency; the hadronic model used in MC simulation.

To estimate the STK track selection efficiency, a pure $\mathrm{H}+\mathrm{He}$ sample is selected by applying an independent charge selection based on the BGO and PSD measurement. The STK track selection 
efficiency is estimated as the ratio between the number of the events that survive the BGO-PSD charge selections and the STK selections over the number of the events that survive BGO-PSD charge selections. On the left of Fig.4, the track selection efficiency as a function of BGO energy is presented. The difference between MC and the data is within $4 \%$ up to $10 \mathrm{TeV}$. The difference will be taken as the systematic uncertainty.

The High Energy Trigger (HET) efficiency is estimated by using the unbiased trigger [6], which is pre-scaled by $1 / 512$ at latitudes within $\pm 20^{\circ}$ and $1 / 2048$ elsewhere. The HET efficiency is evaluated as the ratio between the number of events that activate both the unbiased and HET triggers over the number of events that activate unbiased trigger. On the right of Fig.4, the HET efficiency as a function of BGO energy is shown. The difference between MC and the data is within $9 \%$.
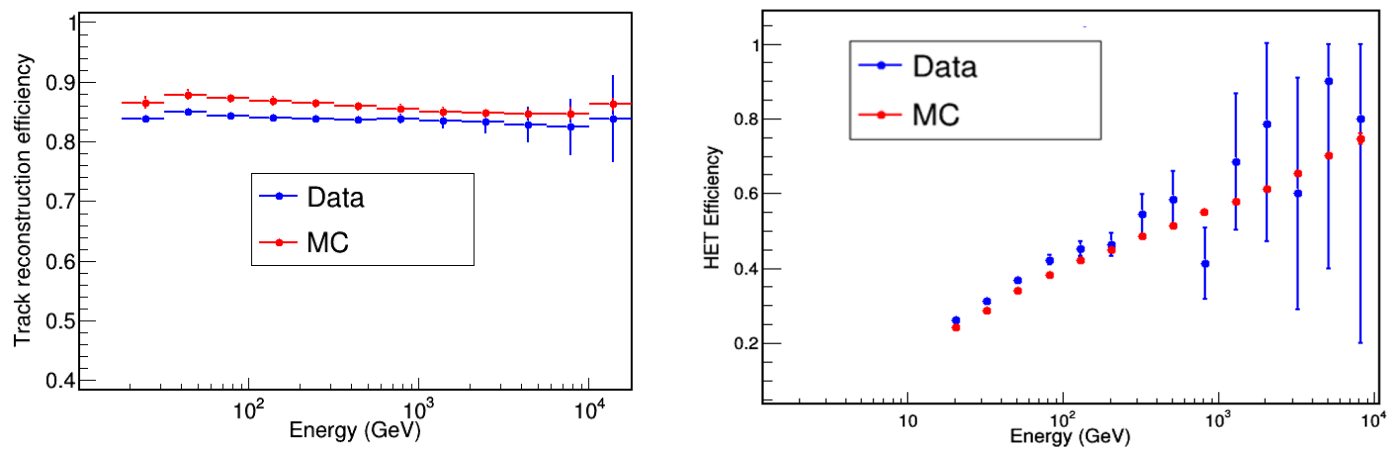

Figure 4: Left: the track selection efficiency as the function of BGO energy. Right: the HET efficiency as the function of BGO energy.

To estimate the charge reconstruction efficiency for one layer of the PSD (layer-1 for instance), we select the $\mathrm{H}+\mathrm{He}$ sample with the layer-2 of the PSD combined with STK charge measurement.The charge reconstruction efficiency as a function of BGO energy is shown in Fig.5. The efficiency differences between the MC and the data are within $4 \%$ up to $10 \mathrm{TeV}$.
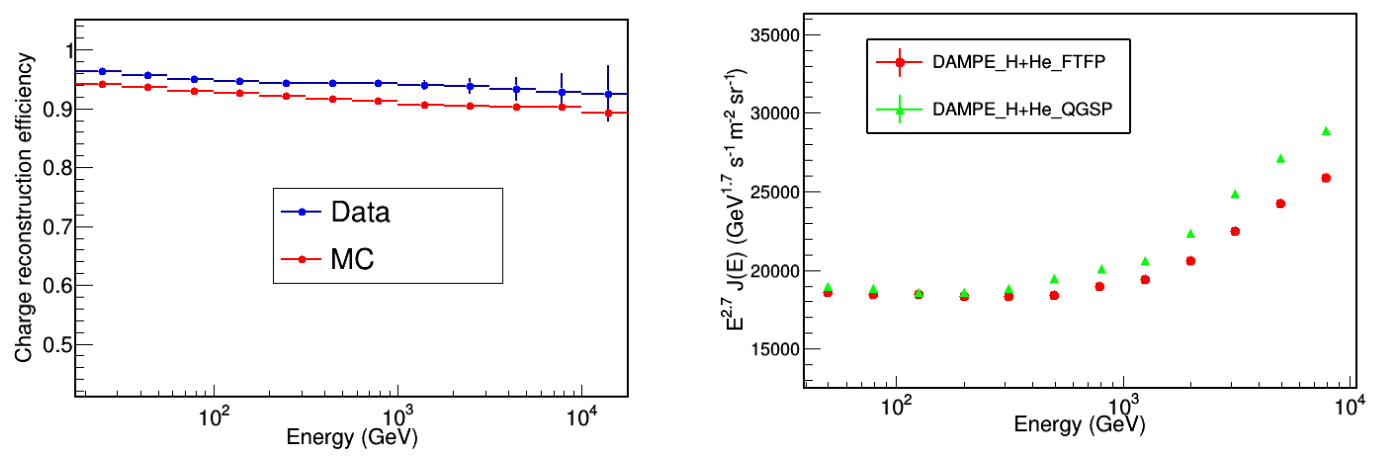

Figure 5: The charge reconstruction efficiency as the function of BGO energy for one PSD layer (left) and the comparison between the two spectra based on two different hadronic models (right).

Due to the limited knowledge of hadronic interactions at high energies, the final result will partially depend on the choice of hadronic model used in the simulation. In this work, the presented 
results are based on the FTFP_BERT (FTFP) model [14]. In addition, the spectrum based on the FTFP_QGSP_BERT (QGSP) model is also computed and compared with spectrum based on the FTFP model to assess the systematic uncertainty. Both spectra based on FTFP and QGSP models respectively are shown on the left of Fig.6. The difference is within 5\% below $1 \mathrm{TeV}$, within $11 \%$ beyond $1 \mathrm{TeV}$.

\section{Results and conclusions}

Fig.6 shows the preliminary $\mathrm{H}+\mathrm{He}$ spectrum measured with DAMPE using $3.8 \times 10^{7} \mathrm{CR}$ events collected from the 1st January 2016 up to the 30th March 2019. The total systematic uncertainty in the flux is $12 \%$ below $1 \mathrm{TeV}$ and $16 \%$ above $1 \mathrm{TeV}$, as shown by the grey shaded area in Fig.6.

The DAMPE H + He spectrum is compared with the ATIC-02 [4], CREAM-III [5], and NUCLEON [19]. Considering the systematic uncertainties, the spectrum is compatible with the previous direct-detection experiments. A clear spectral hardening can be observed below $1 \mathrm{TeV}$ (energy per nucleus), which is in agreement with previously observed hardening at $\sim 250 \mathrm{GeV} / \mathrm{n}$, measured by other experiments separately for $\mathrm{H}, \mathrm{He}$ and heavier cosmic ray nuclei [1] [2] [3]. The extension of the measurement up to $100 \mathrm{TeV}$ is currently ongoing.

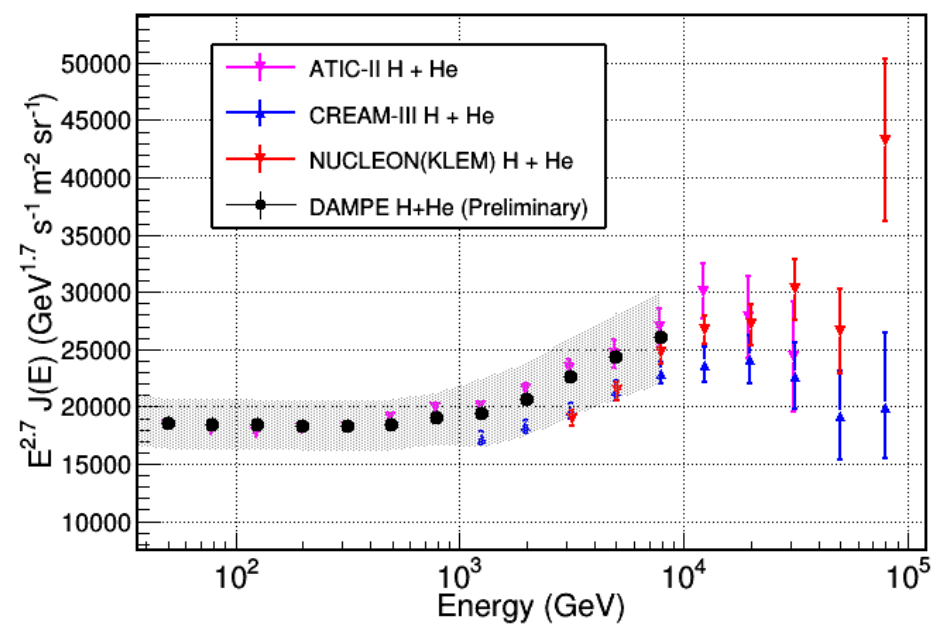

Figure 6: The $\mathrm{H}+\mathrm{He}$ spectrum measured with DAMPE compared with other experiments

\section{Acknowledgments}

The DAMPE mission is funded by the strategic priority science and technology projects in space science of Chinese Academy of Sciences. In China the data analysis is supported in part by the National Key Research and Development Program of China (No. 2016YFA0400200), the National Natural Science Foundation of China (Nos. 11525313, 11622327, 11722328, U1738205, U1738207, U1738208), the strategic priority science and technology projects of Chinese Academy of Sciences (No. XDA15051100), and the 100 Talents Program of Chinese Academy of Sciences. 
In Europe the activities and the data analysis are supported by the Swiss National Science Foundation (SNSF), Switzerland, and the National Institute for Nuclear Physics (INFN), Italy.

\section{References}

[1] O. Adriani et al. [PAMELA Collaboration], PAMELA Measurements of Cosmic-ray Proton and Helium Spectra, Science 332 (2011) 69 [arXiv: 1103.4055].

[2] M. Aguilar et al. [AMS Collaboration], Precision Measurement of the Proton Flux in Primary Cosmic Rays from Rigidity 1 GV to 1.8 TV with the Alpha Magnetic Spectrometer on the International Space Station, Phys. Rev. Lett. 114 (2015) 171103.

[3] O. Adriani et al. [CALET Collaboration], Direct Measurement of the Cosmic-Ray Proton Spectrum from $50 \mathrm{GeV}$ to $10 \mathrm{TeV}$ with the Calorimetric Electron Telescope on the International Space Station, Phys. Rev. Lett. 122 (2019) 181102.

[4] A. D. Panov et al., Energy Spectra of Abundant Nuclei of Primary Cosmic Rays from the Data of ATIC-2 Experiment: Final Results, Bull. Russ. Acad. Sci. Phys. 73 (2009) 564 [arXiv: 1101.3246].

[5] Y. S. Yoon et al., Proton and Helium Spectra from the CREAM-III Flight, Astrophys. J. 839 (2017) no.1, 5 [arXiv: 1704.02512].

[6] J. Chang et al. [DAMPE Collaboration], The Dark Matter Particle Explorer mission, Astropart. Phys. 95 (2017) 6-24 [arXiv: 1706.08453].

[7] Y. H. Yu et al., The Plastic Scintillator Detector at DAMPE, Astropart. Phys. 94 (2017) 1-10 [arXiv: 1703.00098].

[8] P. Azzarelloa, et al., The DAMPE silicon-tungsten tracker, Nucl. Instrum. Meth. A. 831 (2016) 378-384.

[9] Z. Zhang, et al., The calibration and electron energy reconstruction of the BGO ECAL of the DAMPE detector, Nucl. Instrum. Meth. A. 836 (2016) 98-104.

[10] G. Ambrosi et al., The on-orbit calibration of DArk Matter Particle Explorer, Astropart. Phys. 106 (2019) 18-34.

[11] G. O. Dickson, et al., Magnetic anomalies in the South Atlantic and ocean floor spreading, Journal of Geophysical Research. 73 (6) (1968) 2087-2100.

[12] Y. Q. Zhang, et al., The On-orbit Performance of DAMPE Trigger System, PoS(ICRC2017) 232

[13] E. Thebault, et al., International Geomagnetic Reference Field: the 12th generation, Earth, Planets and Space. 67 (2015) 67-79.

[14] https://indico.cern.ch/event/232125/contributions/1541450/attachments/386057/537020/FTF.pdf

[15] A.B. Kaidalov et al., Predictions of Quark-Gluon String Model for pp at LHC, Eur. Phys. J. C 67 (2010) 397-404.

[16] Y. Zhang et al., PSD performance and charge reconstruction with DAMPE, POS(ICRC2017)168.

[17] P. X. Ma et al., A Method of Alignment of the Plastic Scintillator Detector of DAMPE, arXiv: 1808.05720..

[18] G. D' Agostini, A multidimensional unfolding method based on Bayes' theorem Nucl. Instrum. Meth. A 362 (1995) 487-498

[19] E. Atkin, et al., First results of the cosmic ray NUCLEON experiment, Jour. Cosmo. Astropa. Phys 1707 (2017) 020. 